\title{
Scalable Context-Sensitive Points-To Analysis using Multi-Dimensional Bloom Filters.
}

\author{
Rupesh Nasre ${ }^{1}$, Kaushik Rajan ${ }^{2}$, R. Govindarajan ${ }^{1}$, Uday P. Khedker ${ }^{3}$. \\ 1 Indian Institute of Science, Bangalore, India \\ ${ }_{2}^{2}$ Microsoft Research, Bangalore, India \\ 3 Indian Institute of Technology, Bombay, India. \\ nasre@csa.iisc.ernet.in, kaushik@msr.microsoft.com, \\ govind@serc.iisc.ernet.in, uday@cse.iitb.ac.in
}

\begin{abstract}
Context-sensitive points-to analysis is critical for several program optimizations. However, as the number of contexts grows exponentially, storage requirements for the analysis increase tremendously for large programs, making the analysis non-scalable. We propose a scalable flow-insensitive context-sensitive inclusion-based points-to analysis that uses a specially designed multi-dimensional bloom filter to store the points-to information. Two key observations motivate our proposal: (i) points-to information (between pointer-object and between pointerpointer) is sparse, and (ii) moving from an exact to an approximate representation of points-to information only leads to reduced precision without affecting correctness of the (may-points-to) analysis. By using an approximate representation a multi-dimensional bloom filter can significantly reduce the memory requirements with a probabilistic bound on loss in precision. Experimental evaluation on SPEC 2000 benchmarks and two large open source programs reveals that with an average storage requirement of $4 \mathrm{MB}$, our approach achieves almost the same precision $(98.6 \%)$ as the exact implementation. By increasing the average memory to $27 \mathrm{MB}$, it achieves precision upto $99.7 \%$ for these benchmarks. Using Mod/Ref analysis as the client, we find that the client analysis is not affected that often even when there is some loss of precision in the points-to representation. We find that the NoModRef percentage is within $2 \%$ of the exact analysis while requiring $4 \mathrm{MB}$ (maximum $15 \mathrm{MB}$ ) memory and less than 4 minutes on average for the points-to analysis. Another major advantage of our technique is that it allows to trade off precision for memory usage of the analysis.
\end{abstract}

\section{Introduction}

Pointer analysis enables many compiler optimization opportunities and remains as one of the most important compiler analyses. For client analyses, both precision and speed of the underlying pointer analysis play a vital role. Several context-insensitive algorithms have been shown to scale well for large programs [1][2][3][4]. However, these algorithms are significantly less precise for real world 
programs compared to their context-sensitive counterparts[5][6][7][8]. Unfortunately, context-sensitive pointer analysis improves precision at the cost of high often unacceptable - storage requirement and analysis time. These large overheads are an artifact of the large number of contexts that a program might have. For example, the SPEC2000 benchmark eon has 19K pointers if we do not consider context information but the number increases to $417 \mathrm{~K}$ pointers if we consider all context-wise pointers. Scaling a context sensitive points-to analysis is therefore a challenging task. Recent research (see Related Work in Section 5) has focused on the scalability aspect of context-sensitive points-to analysis and achieves moderate success in that direction[9][4]. However, the memory requirements are still considerably large. For instance, in [9], most of the larger benchmarks require over $100 \mathrm{MB}$ for points-to analysis. Hence, scalability still remains an issue. Also, none of the current analyses provide a handle to the user to control the memory usage of a points-to analysis. Such a feature will be useful when analyzing a program in a memory constrained environment.

The objective of a context-sensitive points-to analysis is to construct, for each pointer and context, a set containing all the memory locations (pointees) that the pointer can point to in that context. This paper proposes a new way of representing points-to information using a special kind of bloom filter[10] that we call a multi-dimensional bloom filter.

A bloom filter is a compact, and approximate, representation (typically in the form of bit vectors) of a set of elements which trades off some precision for significant savings in memory. It is a lossy representation that can incur false positives, i.e., an element not in the set may be answered to be in the set. However, it does not have false negatives, i.e., no element in the set would be answered as not in the set. To maintain this property, the operations on a bloom filter are restricted so that items can only be added to the set but can never be deleted ${ }^{1}$. Our motivation for using bloom filters for context-sensitive flowinsensitive points to analysis stems from the following three key observations.

- Conservative static analysis: As with any other compiler analysis, static points-to analysis tends to be conservative as correctness is an absolute requirement. Thus, in case of static may-points-to analysis, a pointer not pointing to a variable at run time can be considered otherwise, but not vice-versa. As a bloom filter does not have false negatives, a representation that uses bloom filters is safe. A bloom filter can only (falsely) answer that a pointer points to a few extra pointees. This only makes the analysis less precise and does not pose any threat to correctness. Further, as a bloom filter is designed to efficiently trade off precision for space it is an attractive representation to enable scalability of points-to analysis.

- Sparse points-to information: The number of pointees that each contextwise pointer (pointer under a given context) actually points to is many orders of magnitude less than both the number of context-wise pointers and the total number of potential pointees. Hence, though the points-to set can

\footnotetext{
${ }^{1}$ Some modified bloom filter structures[11] have been proposed that can support deletion but they do so at the expense of introducing false negatives.
} 
potentially be very large, in practice, it is typically small and sparse. A bloom filter is ideally suited to represent data of this kind. When the set is sparse, a bloom filter can significantly reduce the memory requirement with a probabilistically low bound on loss in precision.

- Monotonic data flow analysis: As long as the underlying analysis uses a monotonic iterative data flow analysis, the size of the points-to set can only increase monotonically. This makes a bloom filter a suitable choice as monotonicity guarantees that there is no need to support deletions.

The above observations make a bloom filter a promising candidate for representing points-to information. However, using the bloom filter as originally proposed in [10] is not efficient for a context sensitive analysis. We therefore extend the basic bloom filter to a multi-dimensional bloom filter (multibloom) to enable efficient storage and manipulation of context aware points-to information. The added dimensions correspond to pointers, calling contexts, and hash functions. The bloom filter is extended along the first two dimensions (pointers and calling contexts) to support all the common pointer manipulation operations $(p=q$, $p=\& q, p=* q$ and $* p=q)$ and the query operation $\operatorname{DoAlias}(p, q)$ efficiently. The third dimension (hash functions) is essential to control loss in precision. We theoretically show and empirically observe that larger the number of hash functions, lower is the loss in precision. In effect, multibloom significantly reduces the memory requirement with a very low probabilistically bound loss in precision. The compact representation of points-to information allows the context sensitive analysis to scale well with the program size.

The major contributions of this paper are:

- We propose a multi-dimensional bloom filter (multibloom) that can compactly represent the points-to information with almost no loss in precision.

- Using extended bloom filter operations, we develop a context-sensitive flowinsensitive points-to analysis for $\mathrm{C}$ programs in the LLVM compilation infrastructure.

- We show that by using multibloom, a user can control the total memory requirement of a compiler analysis, unlike in most other analyses.

- We demonstrate the effectiveness of multibloom through experimental evaluation on 16 SPEC 2000 benchmarks and 2 real world applications. With less than $4 \mathrm{MB}$ memory on average (maximum 15MB), multibloom achieves more than $98 \%$ precision, taking less than 4 minutes per benchmark on average.

- We also evaluate precision of a client Mod/Ref analysis. We find that using multibloom, the NoModRef percentage is within $1.3 \%$ of the exact analysis while requiring $4 \mathrm{MB}$ memory and 4 minutes on average for the points-to analysis.

\section{Background}

General purpose languages like $\mathrm{C}$ pose many challenges to the compiler community. Use of pointers hinders many compiler optimizations. Pointers with multiple 
analysis will be a few orders of magnitude higher than a context insensitive analysis.

Our goal, in this paper, is to reduce this storage and execution time requirement of a context-sensitive points-to analysis. This is achieved by using a variant of bloom filter, which sacrifices a small amount of precision. As we shall see in the next subsection, once the user fixes the size of a bloom filter, he/she can estimate a probabilistic bound on the loss in precision as a function of the average number of pointees of a pointer (in a given context).

\subsection{Bloom Filter}

A bloom filter is a probabilistic data structure used to store a set of elements and test the membership of a given element[10]. In its simplest form, a bloom filter is an array of $N$ bits. An element $e$ belonging to the set is represented by setting the $k$ th bit to 1 , where $h(e)=k$ and $h$ is the hash function mapping element $e$ to $k^{t h}$ bit. For instance, if the hash function is $h_{1}(e)=(3 * e+5) \% N$, and if $N=10$, then for elements $e=13$ and 100 , the bits 4 and 5 are set. Membership of an element $e$ is tested by using the same hash function. Note that element 3 also hashes to the same location as 13 . This introduces false positives, as the membership query would return true for element 3 even if it is not inserted. Note, however, that there is no possibility of false negatives, since we never reset any bit.

The false positive rate can be reduced drastically by using multiple hash functions. Thus, if we use two hash functions for the above example, with $h_{2}(e)=(\lfloor e / 2\rfloor+9) \% N$, then the elements $e=13,100$ get hashed to bits 5,9 . Note that a membership query to 3 would return false as location 0 (corresponding to $\left.h_{2}(3)\right)$ is 0 , even though location 4 (corresponding to $\left.h_{1}(3)\right)$ is set. Thus, using multiple hash functions the false positives can be reduced.

The false positive rate $P$ for a bloom filter of size $N$ bits after $n$ elements are added to the filter with $d$ hash functions is given by Equation 1 (from [10])

$$
P=\frac{(1 / 2)^{d}}{\left(1-\frac{n d}{N}\right)}
$$

This is under the assumption that the individual hash functions are random and different hash functions are independent. Unlike traditional data structures used in points-to analysis[5][8], time to insert elements in a bloom filter and to check for their membership is independent of the number of elements in the filter.

\section{Points-to Analysis using Bloom Filters}

A points-to tuple $\langle p, c, x\rangle$ represents a pointer $p$ pointing to variable $x$ in calling context $c$. A context is defined by a sequence of functions and their call-sites. A naive implementation stores context-sensitive points-to tuples in a bloom filter by hashing the tuple $\langle p, c, x\rangle$ and setting that bit in the bloom filter. This simple 
operation takes care of statements only of the form $p=\& x$. Other pointer statements, like $p=q, p=* q$, and $* p=q$ require additional care. For example, for handling $p=q$ type of statements, the points-to set of $q$ has to be copied to $p$. While bloom filter is very effective for existential queries, it is inefficient for universal queries like "what is the points-to set of pointer $p$ under context $c$ ?".

One way to solve this problem is to keep track of the set of all pointees (objects). This way, the query FindPointsTo $(p, c)$ to find the points-to set for a pointer $p$ under context $c$ is answered by checking the bits that are set for each of the pointees. Although this is possible in theory, it requires storing all possible pointees, making it storage inefficient. Further, going through all of them every time to process a $p=q$ operation makes this strategy time inefficient. Further complications arise if we want to support a context-sensitive DoAlias query. Therefore, we propose an alternative design that has more dimensions than a conventional bloom filter in order to support the pointer operations.

\subsection{Multi-Dimensional Bloom Filter}

Our proposed multi-dimensional bloom filter (multibloom) is a generalization of the basic bloom filter introduced in Section 2.2. It has 4 dimensions, one each for pointers, contexts, hash functions and a bit vector along the fourth dimension. It is represented as $m b[P][C][D][B]$. The configuration of a multibloom is specified by a 7-tuple $\left\langle P, C, D, B, M_{p}, M_{c}, H\right\rangle$ where $P$ is the number of entries for pointers, $C$ is the number of entries for contexts, $D$ is the number of hash functions, $B$ is the bit-vector size for each hash function, $M_{p}$ is the function mapping pointers, $M_{c}$ is the function mapping contexts and $H$ is the family of hash functions. The first 4 entries $(P, C, D, B)$ denote the number of unique values that can be taken along each dimension. For example $C=16$ would mean that the multibloom has space for storing the pointee set for 16 contexts in which a pointer is accessed. We will have to map every context of a given pointer to one among 16 entries. The total size of the structure is Size $=P \times C \times D \times B$. Functions $M_{p}$ and $M_{c}$ map the pointer $p$ and context $c$ to integers $P i d x$ and $C i d x$ in the range $[0, P-1]$ and $[0, C-1]$ respectively. A family of hash functions $H=\left(h_{1}, h_{2}, \cdots, h_{D}\right)$ map the pointee $x$ to $D$ integers $H i d x_{1}, H i d x_{2}, \cdots, H i d x_{D}$ respectively. These play the same role as the hash functions in Section 2.2.

Given a points-to tuple $\langle p, c, x\rangle$, it is entered into the multibloom as follows. Pidx, Cidx and (Hidx $\left.x_{1}, H_{i d x_{2}}, \cdots, H i d x_{D}\right)$ are obtained using $M_{p}, M_{c}$ and $H$ respectively. The tuple is added to multibloom by setting the following $D$ bits:

$$
m b[\text { Pidx }][\mathrm{Cidx}][i]\left[\mathrm{Hidx}_{i}\right]=1, \forall i \in[1, D]
$$

Extending Bloom Filter Operations for $p=q$ Statement: While processing $p=q$ type of statement under context $c$, all we need to do is to find the $B \times D$ source bits from the multibloom that correspond to pointer $q$ under the context $c$ and bitwise-OR it with the $B \times D$ destination bits corresponding to pointer $p$ under context $c$. This logically copies the pointees of $q$ on to $p$ without having to universally quantify all the pointees that $q$ points to. The pseudo-code is given in Algorithm 1. 


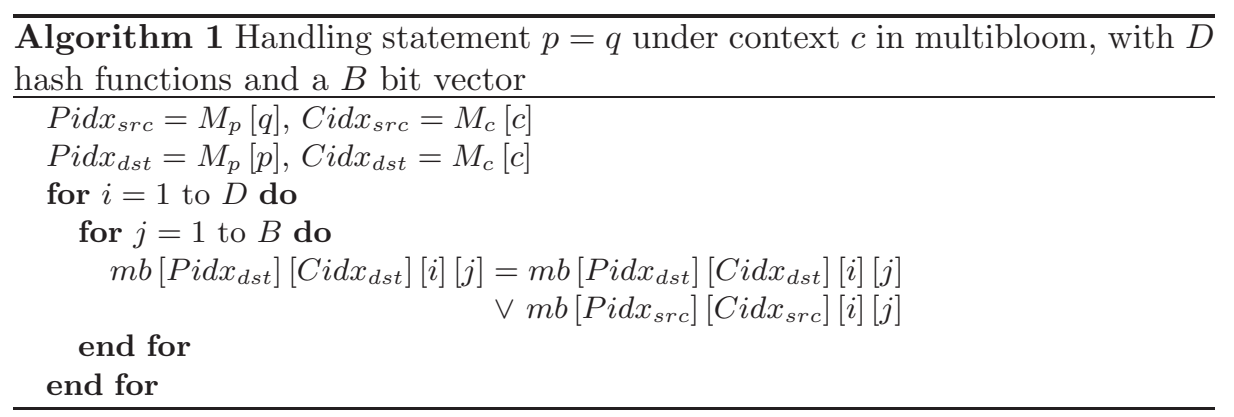

\begin{tabular}{|c|c|c|c|c|c|c|c|c|c|}
\hline statement & \multicolumn{4}{|c|}{ iteration 1} & \multicolumn{5}{|c|}{ iteration 2} \\
\hline \multirow{2}{*}{$\mathrm{p} 3=\mathrm{p} 1$} & \multirow[b]{2}{*}{ p3 } & & & h1 & \multirow{2}{*}{\multicolumn{2}{|c|}{\begin{tabular}{l|l} 
p3 1 \\
\end{tabular}}} & 1 & & hl \\
\hline & & & & h2 & & & 1 & 1 & $\mathrm{~h} 2$ \\
\hline \multirow[b]{2}{*}{$\mathrm{p} 2=\mathrm{p} 3$} & \multirow[b]{2}{*}{$\mathrm{p} 2$} & & & h1 & \multirow{2}{*}{$\mathrm { p } 2 \longdiv { 1 }$} & 1 & 1 & & h1 \\
\hline & & & & h2 & & & 1 & 1 & h2 \\
\hline \multirow[t]{2}{*}{$\mathrm{p} 1=\& \mathrm{x}$} & \multirow[t]{2}{*}{ p1 1} & & & \multirow{2}{*}{$\mid \begin{array}{l}\mathrm{h} 1 \\
\mathrm{~h} 2\end{array}$} & \multirow{2}{*}{\multicolumn{3}{|c|}{ No change. }} & & \\
\hline & & & 1 & & & & & & \\
\hline \multirow{2}{*}{$\mathrm{p} 2=\& \mathrm{y}$} & \multirow{2}{*}{$\mathrm{p} 2$} & $1 \mid$ & & \multirow{2}{*}{$\begin{array}{l}\mathrm{h} 1 \\
\mathrm{~h} 2\end{array}$} & \multirow{2}{*}{\multicolumn{3}{|c|}{ No change. }} & & \\
\hline & & 1 & & & & & & & \\
\hline \multirow[b]{2}{*}{$\mathrm{p} 3=\mathrm{p} 2$} & & $1 \mid$ & & h1 & \multirow{2}{*}{\multicolumn{5}{|c|}{ No change. }} \\
\hline & $\mathrm{p} 3$ & 1 & & h2 & & & & & \\
\hline
\end{tabular}

Fig. 2. Example program to illustrate points-to analysis using bloom filters. First column shows the program statements. Later columns show the state of bloom filters for different pointers after successive iterations over constraints until a fix-point is reached.

Example: Consider the program fragment given in the first column of Figure 2. Consider a multibloom with configuration

$$
\left\langle P, C, D, B, M_{p}, M_{c}, H\right\rangle=\left\langle 3,1,2,8, I, C_{0},\left(h_{1}, h_{2}\right)\right\rangle
$$

The map $M_{p}$ is an identity function $I$ that returns a different value for $p 1, p 2$ and $p 3$. The two hash functions $h_{1}$ and $h_{2}$ are defined as $h_{1}(x)=0, h_{2}(x)=$ $5, h_{1}(y)=3$ and $h_{2}(y)=3 . C_{0}$ maps every context to entry 0 , since $C=1$. As there is only one entry for context and each statement modifies one pointer, we illustrate the multibloom as 3 bloom filters. For clarity, we depict the multibloom as multiple 2-dimensional arrays in Figure 2. Initially, all the bits in the buckets of each pointer are set to 0 . The state of bloom filters after every iteration (the analysis is flow-insensitive) for the example code is shown in Figure 2.

Extending Bloom Filter Operations for $* p=q$ and $p=* q$ : There are two ways to handle statements of the form $* p=q$ and $p=* q$. One way is to extend the above strategy by adding more dimensions to the multibloom. This is extensible to multiple levels of indirection. This strategy would add more dimensions to our 4-dimensional bloom filter, one for each pointer dereference. 
Clearly, this adds to storage and analysis time requirements. The second way is to conservatively assume that a pointer to a pointer points to the universal set of pointees and process the statement conservatively. The number of pointers to pointers is much less in programs compared to single-level pointers. Therefore, depending on the application, one may be willing to lose some precision by this conservative estimate. To obtain a good balance of storage requirement, analysis time and precision, we employ a combination of the above two techniques. We extend multibloom for two-level pointers $\left({ }^{* *} \mathrm{p}\right)$ and use the conservative strategy (universal set of pointees) for higher-level pointers $\left(* * * p,{ }^{* * * *}\right.$ p and so on). The conservative strategy results in little precision loss considering that less than $1 \%$ of all dynamic pointer statements contain more than two levels of pointer indirections (obtained empirically).

Extending multibloom for two-level pointers makes it look like $m b[P][S][C][D][B]$ where $S$ is the number of entries for pointers that are pointees of a two-level pointer. For single-level pointers, $S$ is 1. For two-level pointers $S$ is configurable. For higher-level pointers $S$ is 1 and an additional bit is set to indicate that the pointer points to the universal set of pointees.

To handle load statement $p=* q$ where $p$ is a single-level pointer and $q$ is a two-level pointer, all the cubes $m b[Q][i]$ (i.e., $C \times D \times B$ bits) corresponding to pointer $q, \forall i=1 . . S$ are bitwise-ORed to get a resultant cube. Note that $S=1$ for the result, i.e., the result is for a single-level pointer. This cube is then bitwise-ORed with that of $p$, i.e., with $m b[P][1]$. This makes $p$ point to the pointees pointed to by all pointers pointed to by $q$.

To handle store statement $* q=p$ where $p$ is a single-level pointer and $q$ is a two-level pointer, the cube $m b[P][1]$ of $p$ is bitwise-ORed with each cube $m b[Q][i]$ of $q, \forall i=1 . . S$. It makes each pointer pointed to by $q$ point to the pointees pointed to by $p$.

Handling context-sensitive load/store statements requires a modification to address-of assignment $p=\& q$. If $p$ is a two-level pointer, then to process the address-of statement in context $c, D \times B$ bits of $q$ are bitwise-ORed with $D \times B$ bits of $p$ in the appropriate hash entry for $q$ (see example below).

For mapping a pointer onto the range $1 . . S$, we need a mapping function $M_{s}$. The multibloom configuration is thus extended to include $S$ and $M_{s}$.

Example: Consider the program fragment given in the first column of Figure 3. Consider a multibloom with configuration

$$
\left\langle P, S, C, D, B, M_{p}, M_{s}, M_{c}, H\right\rangle=\left\langle 5,2,1,1,8, I, h_{s},-,(h)\right\rangle
$$

The map $M_{p}$ is an identity function $I$ that returns a different value for $p 1$ through $p 5$ The hash function $h$ is defined as $h(x)=1$ and $h(y)=4$. The mapping function $h_{s}$ is defined as $h_{s}(p 1)=1$ and $h_{s}(p 2)=2$. Initially, all bits in the buckets for each pointer are set to 0 . The state of bloom filters after each statement is processed is shown in the second column of Figure 3. Third column describes the multibloom operation. Note that the above strategy of using an additional dimension for two-level pointers can be extended to include more dimensions to accommodate higher-level pointers. 


\begin{tabular}{|c|c|c|c|c|}
\hline statement & \multicolumn{3}{|c|}{ multibloom processing. } & comments. \\
\hline $\mathrm{p} 1=\& \mathrm{x}$ & $\mathrm{p} 1$ & 1 & & set bit 1 corresponding to $x$. \\
\hline $\mathrm{p} 2=\& \mathrm{y}$ & $\mathrm{p} 2$ & & 1 & set bit 4 corresponding to $y$. \\
\hline $\mathrm{p} 3=\& \mathrm{p} 1$ & p3 & 1 & & bitwise-OR p1's bucket. \\
\hline & & & & \\
\hline $\mathrm{p} 4=\& \mathrm{p} 2$ & $\mathrm{p} 4$ & & 1 & bitwise-OR p2's bucket. \\
\hline $\mathrm{p} 3=\mathrm{p} 4$ & p3 & 1 & & bitwise-OR corresponding \\
\hline & p5 & & 1 & buckets of $\mathrm{p} 3$ and $\mathrm{p} 4$. \\
\hline $\mathrm{p} 5=* \mathrm{p} 3$ & $\mathrm{p} 5$ & 1 & 1 & $\begin{array}{l}\text { bitwise-OR p3's buckets, } \\
\text { bitwise-OR with p5's bucket. }\end{array}$ \\
\hline
\end{tabular}

Fig. 3. Example program to illustrate handling load/store statements. First column shows the program statements. Second column shows the bloom filter state after each statement is processed. Third column describes the multibloom operation.

Storage Requirement of Multibloom: A quick analysis explains why multibloom is space efficient. Consider the SPEC 2000 benchmark parser which has about $10 \mathrm{~K}$ pointers and an average of 3 pointees per context-wise pointer, on an average about 16 contexts per pointer, and around $20 \%$ two- or higherlevel runtime pointer-statements. Consider a multibloom with $P=10 \mathrm{~K}, S=5$, $C=8, D=8$ and $B=50$. The total memory requirement for the multibloom is $10 \mathrm{~K} \times(0.2 \times 5+0.8 \times 1) \times 8 \times 8 \times 50$ bits $=4.32 \mathrm{MB}$. This is much less than what a typical analysis would require, which is at least a few tens of megabytes for a program having $10 \mathrm{~K}$ pointers.

To measure the false positive rate we will now try to map the values back from a 4-dimensional multibloom to a 2-dimensional bloom filter so that we can apply Equation 1 . As there are 16 contexts on an average per pointer and $C=8$, on average 2 contexts would map to a given context bin. Therefore the number of entries per bloom filter would be twice the average number of pointees per context-wise pointer. Now assuming the representation across pointers is more or less uniform, we can use the equation with $N=B \times D=400, d=D=8, n=$ $3 \times 2=6$ (average number of contexts per bin multiplied by average number of pointees per context-wise pointer). This gives a false positive rate of $0.5 \%$ per Equation 1. In practice we find that the loss in precision is not perceivable at all. The NoAlias percentage, a metric used in [13] (explained in Section 4), in this case for the approximate representation is exactly the same as that for an exact representation which takes significantly higher amounts of memory.

\subsection{Querying the Multibloom}

The ultimate goal of alias analysis is to answer whether two pointers $p$ and $q$ alias with each other either in a specific calling context or in a context-insensitive manner. We describe below how multibloom can be used to answer these queries. 

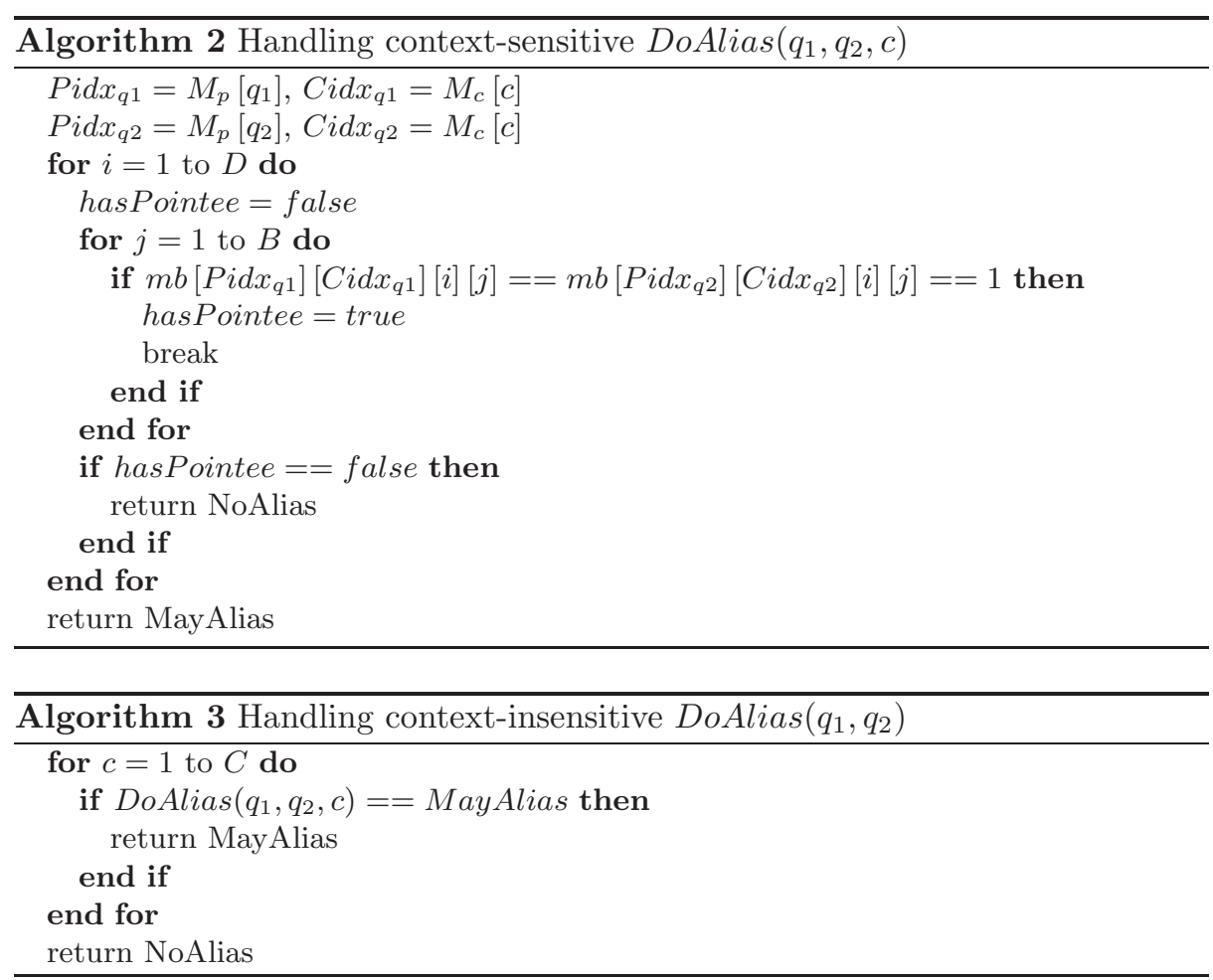

Context-Sensitive Query A context-sensitive query is of type $\operatorname{DoAlias}\left(q_{1}, q_{2}, c\right)$. To answer this query we need to first extract the $B \times D$ bit sets that belong to $q_{1}$ and $q_{2}$ under the context $c$. For each hash function the algorithm needs to determine if the corresponding bit vectors have at least one common bit with the value 1 . If no such bit exists for any one hash function, then $q_{1}$ and $q_{2}$ do not alias. The pseudo-code is given in Algorithm 2. Note that this procedure is for single level pointers. In case $q_{1}$ and $q_{2}$ are higher-level pointers, the outermost for-loop of the procedure needs to be run for each value of $s$ where $s \in[1 . . S]$.

Context-Insensitive Query A context-insensitive query will be of type $\operatorname{DoAlias}\left(q_{1}, q_{2}\right)$. The query is answered by iterating over all possible values of the context $c$ and calling the context-sensitive version of DoAlias: DoAlias $\left(q_{1}, q_{2}, c\right)$. Only if under no context do $q_{1}$ and $q_{2}$ alias, it concludes that there is no alias. The pseudo-code is shown in Algorithm 3.

\section{Experimental Evaluation.}

\subsection{Implementation Details and Experimental Setup}

All our implementation is done in the LLVM compiler infrastructure[13] and the analysis is run as a post linking phase. We implement two points-to analyses, one 
which has an exact representation (without false positives) of the points-to set and the other uses our proposed multiblooom representation. For an exact representation we store pointees per context for a pointer using STL vectors[14]. Both versions are implemented by extending Andersen's algorithm [15] for contextsensitivity. They are flow-insensitive and field-insensitive implementations that use an invocation graph based approach. Each aggregate (like arrays and structures) is represented using a single memory location. Neither version implements optimizations like offline variable substitution[16].

\begin{tabular}{|l|r|r|r|r|}
\hline $\begin{array}{l}\text { Bench } \\
\text {-mark }\end{array}$ & KLOC & $\begin{array}{l}\text { Total } \\
\text { Inst }\end{array}$ & $\begin{array}{c}\text { Pointer } \\
\text { Inst }\end{array}$ & $\begin{array}{r}\text { No. of } \\
\text { Fns }\end{array}$ \\
\hline gcc & 222.185 & 328425 & 119384 & 1829 \\
perlbmk & 81.442 & 143848 & 52924 & 1067 \\
vortex & 67.216 & 75458 & 16114 & 963 \\
eon & 17.679 & 126866 & 43617 & 1723 \\
httpd & 125.877 & 220552 & 104962 & 2339 \\
sendmail & 113.264 & 171413 & 57424 & 1005 \\
parser & 11.394 & 35814 & 11872 & 356 \\
gap & 71.367 & 118715 & 39484 & 877 \\
vpr & 17.731 & 25851 & 6575 & 228 \\
crafty & 20.657 & 28743 & 3467 & 136 \\
mesa & 59.255 & 96919 & 26076 & 1040 \\
ammp & 13.486 & 26199 & 6516 & 211 \\
twolf & 20.461 & 49507 & 15820 & 215 \\
gzip & 8.618 & 8434 & 991 & 90 \\
bzip2 & 4.650 & 4832 & 759 & 90 \\
mcf & 2.414 & 2969 & 1080 & 42 \\
equake & 1.515 & 3029 & 985 & 40 \\
art & 1.272 & 1977 & 386 & 43 \\
\hline
\end{tabular}

Table 1. Benchmark characteristics

\begin{tabular}{|l|r|r|r|r|}
\hline Bench & \multicolumn{2}{|c|}{ Memory (KB) } & \multicolumn{2}{|c|}{ Precision } \\
\cline { 2 - 5 } -mark & \multicolumn{1}{|c|}{$S=1$} & \multicolumn{1}{|c|}{$S=5$} & $S=1$ & $S=5$ \\
\hline gcc & 220973 & 302117.00 & 84.2 & 85.3 \\
perlbmk & 99346.9 & 143662.00 & 89.3 & 90.6 \\
vortex & 44756.4 & 62471.00 & 91 & 91.5 \\
eon & 108936 & 131552.00 & 96.3 & 96.8 \\
httpd & 221633 & 233586.00 & 92.8 & 93.2 \\
sendmail & 122310 & 127776.00 & 90.2 & 90.4 \\
parser & 23511.4 & 43093.10 & 97 & 98 \\
gap & 74914.8 & 84551.70 & 96.7 & 97.4 \\
vpr & 15066.4 & 23676.60 & 93.6 & 94.2 \\
crafty & 10223.9 & 10891.20 & 96.9 & 97.6 \\
mesa & 50389.7 & 55066.90 & 99.2 & 99.4 \\
ammp & 12735.8 & 15282.90 & 99.1 & 99.2 \\
twolf & 29037.2 & 33663.10 & 99.1 & 99.3 \\
gzip & 2807 & 3005.9 & 90.6 & 90.9 \\
bzip2 & 2128.51 & 2333.82 & 87.7 & 88 \\
mcf & 2122.09 & 3758.17 & 94.5 & 94.5 \\
equake & 2245.6 & 3971.50 & 97.6 & 97.7 \\
art & 1090.72 & 1693.82 & 88.6 & 88.6 \\
\hline
\end{tabular}

Table 2. Sensitivity to parameter $S$.

We evaluate performance over $16 \mathrm{C} / \mathrm{C}++$ SPEC 2000 benchmarks and two large open source programs: $h t t p d$ and sendmail. Their characteristics are given in Table 1. KLOC is the number of Kilo lines of code, Total Inst is the total number of static LLVM instructions, Pointer Inst is the number of static pointer-type LLVM instructions and No. of Fns is the number of functions in the benchmark. The LLVM intermediate representations of SPEC 2000 benchmarks and open source programs were run using opt tool of LLVM on an Intel Xeon machine with $2 \mathrm{GHz}$ clock, $4 \mathrm{MB}$ L2 cache and $3 \mathrm{~GB}$ RAM. To quantify the loss in precision with a multibloom implementation, we use the NoAlias percentage metric used in LLVM. It is calculated by making a set of alias queries for all pairs of pointer variables within each function in a program and counting the number of queries that return NoAlias. Larger the NoAlias percentage, more precise is the analysis (upper bounded by the precision of the exact analysis).

We evaluate the performance of a multibloom for many different configurations and compare it with the exact implementation. In all evaluated configurations we allow the first dimension $(P)$ to be equal to the number of unique pointers. We empirically found that the number of entries $S$ for pointers pointed 


\begin{tabular}{|c|c|c|c|c|c|c|c|c|c|c|}
\hline \multirow{3}{*}{$\begin{array}{l}\text { Bench } \\
\text {-mark }\end{array}$} & \multicolumn{5}{|c|}{ Precision (NoAlias \%) } & \multicolumn{5}{|c|}{ Memory (KB) } \\
\hline & \multirow[b]{2}{*}{ exact } & \multicolumn{4}{|c|}{ multibloom } & \multirow[b]{2}{*}{ exact } & \multicolumn{4}{|c|}{ multibloom } \\
\hline & & $\begin{array}{l}4-4-10 \\
\text { tiny }\end{array}$ & $\begin{array}{r}8-8-10 \\
\text { small }\end{array}$ & \begin{tabular}{|c|}
$8-12-50$ \\
medium
\end{tabular} & $\begin{array}{c}8-16-100 \\
\text { large }\end{array}$ & & $\begin{array}{c}4-4-10 \\
\text { tiny }\end{array}$ & $\begin{array}{r}8-8-10 \\
\text { small }\end{array}$ & \begin{tabular}{|c|}
$8-12-50$ \\
medium
\end{tabular} & $\begin{array}{c}8-16-100 \\
\text { large }\end{array}$ \\
\hline gcc & OOM & 71.8 & 79.6 & 83.4 & 85.3 & $\mathrm{OOM}$ & 3956 & 15445 & 113577 & 302117 \\
\hline perlbmk & OOM & 75.3 & 85.0 & 89.3 & 90.6 & OOM & 1881 & 7345 & 54008 & 143662 \\
\hline vortex & OOM & 85.7 & 90.1 & 91.2 & 91.5 & OOM & 818 & 3194 & 23486 & 62471 \\
\hline eon & 96.8 & 81.5 & 88.9 & 94.3 & 96.8 & 385284 & 3059 & 11942 & 87814 & 233586 \\
\hline httpd & 93.2 & 90.1 & 92.1 & 92.9 & 93.2 & 225513 & 1673 & 6533 & 48036 & 127776 \\
\hline sendmail & 90.4 & 85.6 & 88.2 & 90.3 & 90.4 & 197383 & 1723 & 6726 & 49455 & 131552 \\
\hline parser & 98.0 & 65.8 & 97.3 & 97.9 & 98.0 & 121588 & 565 & 2204 & 16201 & 43094 \\
\hline p & 97.5 & 88.2 & 93.5 & 96.7 & 97.4 & 97863 & 1107 & 4323 & 31786 & 84552 \\
\hline vpr & 94.2 & 85.9 & 93.9 & 94.1 & 94.2 & 50210 & 310 & 1211 & 8901 & 23677 \\
\hline crafty & 97.6 & 97.1 & 97.6 & 97.6 & 97.6 & 15986 & 143 & 557 & 4095 & 10892 \\
\hline mesa & 99.4 & 89.6 & 96.6 & 99.1 & 99.4 & 8261 & 721 & 2816 & 20702 & 55067 \\
\hline ammp & 99.2 & 98.4 & 99.0 & 99.2 & 99.2 & 5844 & 201 & 782 & 5746 & 15283 \\
\hline twolf & 99.3 & 96.7 & 99.1 & 99.3 & 99.3 & 1594 & 441 & 1721 & 12656 & 33664 \\
\hline gzip & 90.9 & 88.8 & 90.5 & 90.8 & 90.9 & 1447 & 42 & 164 & 1205 & 3205 \\
\hline bzip2 & 88.0 & 84.8 & 88.0 & 88.0 & 88.0 & 519 & 31 & 120 & 878 & 2334 \\
\hline & 94.5 & 91.3 & 94.3 & 94.5 & 94.5 & 220 & 50 & 193 & 1413 & 3759 \\
\hline equake & 97.7 & 96.9 & 97.7 & 97.7 & 97.7 & 161 & 52 & 204 & 1494 & 3972 \\
\hline art & 88.6 & 86.6 & 88.4 & 88.6 & 88.6 & 42 & 23 & 87 & 637 & 1694 \\
\hline
\end{tabular}

Table 3. Precision (NoAlias \%) vs Memory (in KB). OOM means Out Of Memory.

to by two-level pointers gives a good trade off between memory and precision for $S=5$. The hash family $H$, the context mapper $M_{c}$ and the pointer-location mapper $M_{s}$ are derived from the in-built pseudo random number generator. Many different combinations were tried for the other three dimensions: $C=(4,8,16)$, $B=(10,20,50,100)$ and $D=(4,8,12,16)$. From now on, when we report the results, we refer to the multibloom configuration by the tuple $(C-D-B)$. Below we report the results for select configurations that showed interesting behavior.

\subsection{Tradeoff between Precision, Memory and Analysis Time}

In Tables 3-4 we report the precision, time and memory requirements for various benchmarks. We compare 4 different multibloom configurations namely tiny $t$ (44-10), small s (8-8-10), medium $m$ (8-12-50) and large $l$ (8-16-100) with exact which does not have any false positives.

Three out of the 18 benchmarks run out of memory when we run an exact analysis, highlighting the need for a scalable context-sensitive points-to analysis. All the multibloom configurations ran to completion successfully for these three benchmarks. The tiny configuration indicates significant reduction in both memory and analysis time. The memory requirement is three orders less, while the access time is reduced to about one-fourth for all benchmarks which take at least 20 seconds. The precision (in terms of NoAlias percentage) is within $7 \%$ for tiny of an exact analysis on average. At the other end, medium and large config- 


\begin{tabular}{|c|c|c|c|c|c|c|c|c|c|c|}
\hline \multirow{3}{*}{$\begin{array}{l}\text { Bench } \\
\text {-mark }\end{array}$} & \multicolumn{5}{|c|}{ Precision (NoAlias \%) } & \multicolumn{5}{|c|}{ Time $(\mathrm{s})$} \\
\hline & \multirow[b]{2}{*}{ exact } & \multicolumn{4}{|c|}{ multibloom } & \multirow[b]{2}{*}{ exact } & \multicolumn{4}{|c|}{ multibloom } \\
\hline & & $\mathrm{t}$ & $\mathrm{s}$ & $\mathrm{m}$ & 1 & & $\mathrm{t}$ & $T$ & $\mathrm{~m}$ & \\
\hline gcc & OOM & 71.8 & 79.6 & 83.4 & 85.3 & OOM. & 791.705 & 3250.627 & 10237.702 & 27291.303 \\
\hline perlbmk & OOM & 75.3 & 85.0 & 89.3 & 90.6 & OOM. & 76.277 & 235.207 & 2632.044 & 5429.385 \\
\hline vortex & OOM & 85.7 & 90.1 & 91.2 & 91.5 & OOM. & 95.934 & 296.995 & 1998.501 & 4950.321 \\
\hline eon & 96.8 & 81.5 & 88.9 & 94.3 & 96.8 & 231.166 & 39.138 & 118.947 & 1241.602 & 2639.796 \\
\hline httpd & 93.2 & 90.1 & 92.1 & 92.9 & 93.2 & 17.445 & 7.180 & 15.277 & 52.793 & 127.503 \\
\hline sendmail & 90.4 & 85.6 & 88.2 & 90.3 & 90.4 & 5.956 & 3.772 & 6.272 & 25.346 & 65.889 \\
\hline parser & 98.0 & 65.8 & 97.3 & 97.9 & 98.0 & 55.359 & 9.469 & 31.166 & 145.777 & 353.382 \\
\hline gap & 97.5 & 88.2 & 93.5 & 96.7 & 97.4 & 144.181 & 5.444 & 17.469 & 152.102 & 419.392 \\
\hline vpr & 94.2 & 85.9 & 93.9 & 94.1 & 94.2 & 29.702 & 5.104 & 18.085 & 88.826 & 211.065 \\
\hline crafty & 97.6 & 97.1 & 97.6 & 97.6 & 97.6 & 20.469 & 2.636 & 9.069 & 46.899 & 109.115 \\
\hline mesa & 99.4 & 89.6 & 96.6 & 99.1 & 99.4 & 1.472 & 1.384 & 2.632 & 10.041 & 23.721 \\
\hline ammp & 99.2 & 98.4 & 99.0 & 99.2 & 99.2 & 1.120 & 1.008 & 2.592 & 15.185 & 38.018 \\
\hline volf & 99.3 & 96.7 & 99.1 & 99.3 & 99.3 & 0.596 & 0.656 & 1.152 & 5.132 & 12.433 \\
\hline gzip & 90.9 & 88.8 & 90.5 & 90.8 & 90.9 & 0.348 & 0.192 & 0.372 & 1.808 & 4.372 \\
\hline bzip2 & 88.0 & 84.8 & 88.0 & 88.0 & 88.0 & 0.148 & 0.144 & 0.284 & 1.348 & 3.288 \\
\hline mcf & 94.5 & 91.3 & 94.3 & 94.5 & 94.5 & 0.112 & 0.332 & 0.820 & 5.036 & 12.677 \\
\hline equake & 97.7 & 96.9 & 97.7 & 97.7 & 97.7 & 0.224 & 0.104 & 0.236 & 1.104 & 2.652 \\
\hline art & 88.6 & 86.6 & 88.4 & 88.6 & 88.6 & 0.168 & 0.164 & 0.408 & 2.404 & 6.132 \\
\hline
\end{tabular}

Table 4. Precision (NoAlias \%) vs Time (in sec). OOM means Out Of Memory. $t$ is tiny, $s$ is small, $m$ is medium and $l$ is large configuration.

urations achieve full precision for all the benchmarks with significant savings in memory requirement for those requiring at least $15 \mathrm{MB}$ memory. However, this comes at a price in terms of analysis time. Thus medium and large are good configuration to use if precision is an absolute requirement. Even for the larger benchmarks they will lead to termination as they still provide a compact storage.

The small configuration proves to be an excellent trade off point. It achieves a good precision (within 1.5\%) on average and achieves more than 10-fold memory reduction for benchmarks requiring more than $10 \mathrm{MB}$ memory for an exact analysis. It takes around the same amount of time on benchmarks that terminate with exact analysis. It should be noted that for smaller benchmarks (mesa, ammp, twolf, gzip, bzip2, mcf, equake and art) the configuration small requires more time than exact configuration. However, for larger benchmarks we see significant improvements in analysis time using bloom filter. One unique advantage of using multibloom is the user-control over various parameters to trade off precision for memory or vice versa. To reduce memory requirement for medium and large, we experimented with smaller values of $S$. The results for $S=1$ versus $S=5$ are given in Table 2 (memory in KB and precision as NoAlias percentage). We observe that with at most $1 \%$ reduction in average precision, we can obtain around $18 \%$ reduction in average memory requirement. In summary, a multibloom representation guarantees a compact storage representation for context-sensitive points-to analysis and allows the user to pick the configuration depending on whether analysis time or accuracy is more desirable. 


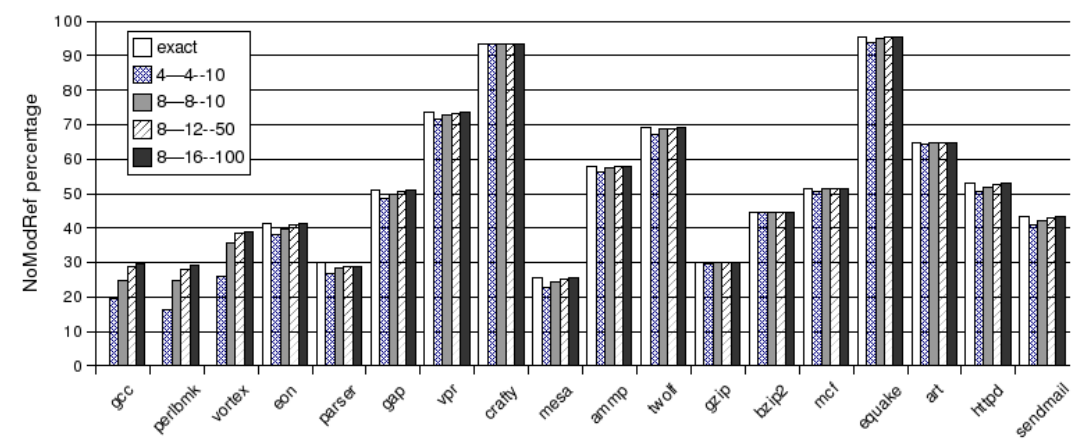

Fig. 4. Mod/Ref client analysis.

\subsection{Mod/Ref Analysis as a Client to Points-to Analysis}

Next we analyze how the loss in precision in the points-to analysis due to false positives affect the client analyses. We use the Mod/Ref analysis as the client of our multibloom based points-to analysis. For a query GetModRef (callsite, pointer), the Mod/Ref analysis checks whether callsite reads or modifies the memory pointed to by pointer. It has four outcomes: (i) NoModRef: call-site does not read from or write to memory pointed to by pointer, (ii) Ref: call-site reads from the memory pointed to by pointer, (iii) Mod: call-site writes to (and does not read from) the memory pointed to by pointer, and (iv) ModRef: call-site reads from and writes to the memory pointed to by pointer. ModRef is most conservative and should be returned when it is not possible to establish otherwise for a safe analysis. The more precise an approximate points-to analysis the more often will it answer NoModRef (upper bounded by an exact analysis). Figure 4 shows percentage of queries answered NoModRef by the analysis. From the figure, it can be seen that the NoModRef percentage with multibloom is $96.9 \%$ of the exact analysis even with a tiny configuration. For small configuration, it improves further to $98.7 \%$. This shows that a client analysis is hardly affected due to loss in precision by using an approximate representation, while still enjoying the benefits of reduced memory and time requirements.

An important aspect of using multibloom is the provision of selecting a configuration on need basis. For more precise analysis, one can trade off memory and speed requirements by choosing larger values for $C, D$ and $B$. For scalable analyses, one can reduce these values trading off some precision.

\section{Related Work.}

Many scalable pointer analysis algorithms are context- and flow-insensitive [1]. As scalability became an important factor with increasing code size, interesting mechanisms were introduced to approximate the precision of a full blown 
context-sensitive and flow-sensitive analysis. [17] proposed one level flow to improve precision of context-insensitive, flow-insensitive analyses, still maintaining the scalability. Later, several inclusion-based scalable analyses were proposed [2][3][4], based on some novel data structures for points-to analysis like BDD. Similar to ours, several context-sensitive but flow-insensitive analyses have been recently proposed. Since inclusion-based analyses are costly, several unificationbased algorithms were introduced, trading off precision for speed [1], [18]. Several context-sensitive algorithms proposed earlier [5][6][7][8] are flow-sensitive. Flowsensitivity adds to precision but typically makes the analysis non-scalable. The idea of bootstrapping [19] enables context- and flow-sensitive algorithms to scale.

Various enhancements have also been made to the original Andersen's inclusionbased algorithm: online cycle elimination[20] to break dependence cycles on the fly, offline variable substitution[16] to reduce the number of pointers tracked during the analysis, location equivalence[21] and semi-sparse flow-sensitivity[22]. These enhancements are orthogonal to the usage of bloom filters. One can implement a points-to analysis with, for instance, online cycle elimination with points-to tuples stored in bloom filters and enjoy combined benefits.

Several novel data structures have been used in the last decade to scale points-to analysis, like ROBDD[2][23][9], ZBDD[24]. These data structures store exact representation of the points-to information and have no false positives. In contrast, bloom filters are useful for storing information in an approximate way. Also, our multibloom filter approach provides the user to control the memory requirement with a probabilistic lower bound on the loss in precision. Optimistic results for pointer analysis hint that bloom filters would be very useful for other compiler analyses as well.

\section{Conclusions}

In this paper we propose the use of multi-dimensional bloom filter for storing points-to information. The proposed representation, though, may introduce false positives, significantly reduces the memory requirement and provides a probabilistic lower bound on loss of precision. As our multibloom representation introduces only false positives, but no false negatives, it ensures safety for (may-)points-to analysis. We demonstrate the effectiveness of multibloom on 16 SPEC 2000 benchmarks and 2 real world applications. With average 4MB memory, multibloom achieves almost the same (98.6\%) precision as the exact analysis taking about average 4 minutes per benchmark. Using Mod/Ref analysis as the client, we find that the client analysis is not affected that often even with some loss of precision in points-to representation. Our approach, for the first time, provides user a control on the memory requirement, yet giving a probabilistic lower bound on the loss in precision. As a future work, it would be interesting to see the effect of approximation introduced using bloom filters with the approximations introduced in control-flow analyses such as kCFA or in unification of contexts. 


\section{References}

1. B. Steensgaard, "Points-to analysis in almost linear time," in $P O P L, 1996$.

2. M. Berndl, O. Lhotak, F. Qian, L. Hendren, and N. Umanee, "Points-to analysis using BDDs," in PLDI, 2003.

3. N. Heintze and O. Tardieu, "Ultra-fast aliasing analysis using CLA: A million lines of C code in a second," in PLDI, 2001.

4. O. Lhotak and L. Hendren, "Scaling Java points-to analysis using spark," in $C C$, 2003.

5. M. Emami, R. Ghiya, and L. J. Hendren, "Context-sensitive interprocedural pointsto analysis in the presence of function pointers," in PLDI, 1994.

6. W. Landi, B. G. Ryder, and S. Zhang, "Interprocedural modification side effect analysis with pointer aliasing," in PLDI, 1993.

7. J. Whaley and M. Rinard, "Compositional pointer and escape analysis for java programs," in OOPSLA, 1999.

8. R. P. Wilson and M. S. Lam, "Efficient context-sensitive pointer analysis for C programs," in PLDI, 1995.

9. J. Whaley and M. S. Lam, "Cloning-based context-sensitive pointer alias analysis using binary decision diagrams," in PLDI, 2004.

10. B. H. Bloom, "Space/time trade-offs in hash coding with allowable errors," in Communications of the ACM 13(7):422426, 1970.

11. L. Fan, P. Cao, J. Almeida, and A. Z. Broder, "Summary cache: a scalable widearea web cache sharing protocol," in SIGCOMM, 1998.

12. R. Rugina and M. Rinard, "Pointer analysis for multithreaded programs," in PLDI, 1999.

13. http://llvm.org, "The LLVM compiler infrastructure,"

14. http://en.wikipedia.org/wiki/Standard_Template_Library, "Standard Template Library,"

15. L. O. Andersen, "Program analysis and specialization for the $\mathrm{C}$ programming language," in PhD Thesis, 1994.

16. A. Rountev and S. Chandra, "Offline variable substitution for scaling points-to analysis," in PLDI, 2000

17. M. Das, "Unification-based pointer analysis with directional assignments," in PLDI, 2000.

18. M. Fahndrich, J. Rehof, and M. Das, "Scalable context-sensitive flow analysis using instantiation constraints," in PLDI, 2000.

19. V. Kahlon, "Bootstrapping: a technique for scalable flow and context-sensitive pointer alias analysis," in PLDI, 2008.

20. M. Faehndrich, J. S. Foster, Z. Su, and A. Aiken, "Partial online cycle elimination in inclusion constraint graphs," in PLDI, 1998.

21. B. Hardekopf and C. Lin, "Exploiting pointer and location equivalence to optimize pointer analysis," in $S A S, 2007$.

22. B. Hardekopf and C. Lin, "Semi-sparse flow-sensitive pointer analysis," in POPL, 2009 .

23. J. Zhu and S. Calman, "Symbolic pointer analysis revisited," in PLDI, 2004.

24. O. Lhotak, S. Curial, and J. N. Amaral, "Using ZBDDs in points-to analysis," in LCPC, 2007. 